Vietnam Journal of Mechanics, VAST, Vol. 34, No. 3 (2012), pp. $139-156$

\title{
SOLVING NONLINEAR STABILITY PROBLEM OF IMPERFECT FUNCTIONALLY GRADED CIRCULAR CYLINDRICAL SHELLS UNDER AXIAL COMPRESSION BY GALERKIN'S METHOD
}

\author{
Dao Van Dung, Le Kha Hoa \\ University of Science, VNU
}

\begin{abstract}
This paper presents an analytical approach to analyze the nonlinear stability of thin closed circular cylindrical shells under axial compression with material properties varying smoothly along the thickness in the power and exponential distribution laws. Equilibrium and compatibility equations are obtained by using Donnel shell theory taking into account the geometrical nonlinearity in von Karman and initial geometrical imperfection. Equations to find the critical load and the load-deflection curve are established by Galerkin's method. Effects of buckling modes, of imperfection, of dimensional parameters and of volume fraction indexes to buckling loads and postbuckling load-deflection curves of cylindrical shells are investigated. In case of perfect cylindrical shell, the present results coincide with the ones of the paper [13] which were solved by Ritz energy method.

Key words: Cylindrical shells, non - linear stability, functionally graded materials, imperfect.
\end{abstract}

\section{INTRODUCTION}

The structures made of functionally graded materials (FGMs) including cylindrical shell structure play an important role in modern industries [1]. Therefore, the research on strength and stability of FGM cylindrical shells are interested very much by scientists. In 2002, Shen [2] solved the postbuckling problem of axially - loaded FGM cylindrical shells in the thermal environments by perturbation technique. By the same method, Shen and Noda [3] analyzed the postbuckling of FGM cylindrical shells under combined axial and radial mechanical loads in the thermal environments. Shahsiah and Eslami [4] based on improved Donnell equations considered FG cylindrical shell themal instability. Wu et al. [5] studied the thermoelastic stability of FG cylindrical shell with the geometrical linearity. Geometrical nonlinear analysis of FG shells was considered by Zhao and Liew [6]. Investigation on buckling of imperfect FG cylindrical shells subjected to axial compression also was presented by Huang and Han [7], but with the linear buckling shape $(\sin (m \pi x / L) \cdot \sin (n y / R))$. Using the 2D higher - order deformation theory, Matsugana [8] solved the problem on free vibration and stability of FG circular cylindrical shells. Shen et al. [9] given the results of postbuckling problem of internal pressure loaded FGM cylindrical shell surrounded by an 
elastic medium according to the third order shear deformation theory. Research on nonlinear postbuckling of eccentrically stiffened FGM plates and shallow shells was reported by Dao Huy Bich et al. [10]. Stability analysis of imperfect FGM cylindrical panels under the mechanical and thermal load, taking into account the geometrical nonlinearity, there were the results of Duc N. D., Tung H. V. [11] and Dao Van Dung, Le Kha Hoa [12].

In recent years (2009 - 2010) Huang and Han [13, 14, 15], by Ritz's method, studied nonlinear elastic buckling and postbuckling of perfect FGM cylindrical shells subjected to axial compressive load, torsion load or radial load. They proposed analytical expression of deflection including the linear buckling shape $(\sin (m \pi x / L) \cdot \sin (n y / R))$ and the nonlinear buckling shape $\left(\sin ^{2}(m \pi x / L)\right)$. This is an interesting different point from the previous papers in literature.

Following this idea, the present paper has developed the results of the paper [13] considering the buckling and postbuckling of axially compressed initial imperfect FGM cylindrical shells with geometrical nonlinearity. In addition, we assume that material properties such as $E$ and $\nu$ change in two distribution laws: Exponential function and Power function of $z$. Applying Galerkin's method have been received the equations for finding the critical buckling load and describing postbuckling load - deflection curve. The effects of geometric parameters, buckling modes, the ratio of volume on buckling load and postbuckling are considered. In case of the perfect cylindrical shell, the gained results return to the ones of [13].

\section{FGM CYLINDRICAL SHELLS AND FUNDAMENTAL EQUATIONS}

\subsection{FGM Cylindrical shells}

Consider a FGM cylindrical thin circular shell with middle surface radius $R$, thickness $h$ and length $L$ (Fig. 1) under axial compression. Assume that two butt - ends of

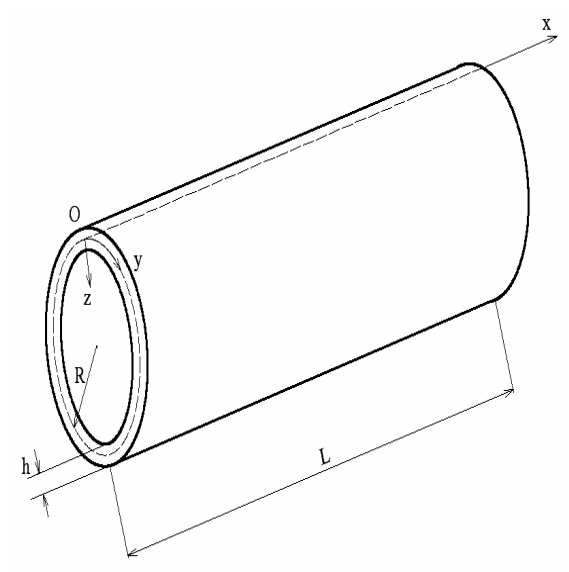

Fig. 1. FGM cylindrical shells

cylindrical shell are only deformed in their planes and they still are circular [16]. The 
FGM cylindrical shell is made from a mixture of ceramic and metal. We choose the cylindrical coordinate $(x, \theta, z) ; y=R \theta$ so that the origin $\mathrm{O}$ is located at the left end and on the middle surface of shell. The coordinate axes $x, y$ and $z$ are respectively in the longitudinal, circumferential and inward radial directions $(-h / 2 \leq z \leq h / 2)$. The Young's modulus and Poisson's ratio of material are assumed to be varied smoothly along the thickness of shell with the power distribution law as [13]

$$
\begin{aligned}
& E=E(z)=E_{m}+\left(E_{c}-E_{m}\right)\left(\frac{2 z+h}{2 h}\right)^{k} \equiv E_{m}+E_{c m} r^{k}, \\
& \nu=\nu(z)=\nu_{m}+\left(\nu_{c}-\nu_{m}\right)\left(\frac{2 z+h}{2 h}\right)^{k_{1}} \equiv \nu_{m}+\nu_{c m} r^{k_{1}}, \\
& E_{c m}=E_{c}-E_{m}, \quad r=\frac{2 z+h}{2 h}, \quad \nu_{c m}=\nu_{c}-\nu_{m}, \quad k \geq 0, \quad k_{1} \geq 0,
\end{aligned}
$$

or with the exponential distribution law as $[17,18]$

$$
E=E(z)=E_{c} e^{-\frac{1}{2}\left(\ln \frac{E_{c}}{E_{m}}\right)\left(1-\frac{2 z}{h}\right)}, \quad \nu=\nu(z)=\nu_{c} e^{-\frac{1}{2}\left(\ln \frac{\nu_{c}}{\nu_{m}}\right)\left(1-\frac{2 z}{h}\right)}
$$

The quantities $E_{m}, E_{c}$ and $\nu_{m}, \nu_{c}$ are Young's moduli and Poisson's ratios corresponding to metal $(\mathrm{m})$ and ceramic $(\mathrm{c})$.

\subsection{Fundamental relations and governing equations}

According to [7, 19], the nonlinear relationship between the strain components on the middle surface of imperfect cylindrical shell with the a deflection in von Karman's sense is as follows

$$
\begin{aligned}
& \varepsilon_{x}^{0}=u_{, x}+\frac{1}{2} w_{, x}^{2}+w_{, x} w_{, x}^{*} \\
& \varepsilon_{y}^{0}=v_{, y}-\frac{w}{R}+\frac{1}{2} w_{, y}^{2}+w_{, y} w_{, y}^{*} \\
& \gamma_{x y}^{0}=u_{, y}+v_{, x}+w_{, x} w_{, y}+w_{, x} w_{, y}^{*}+w_{, y} w_{, x}^{*}
\end{aligned}
$$

where $u=u(x, y), v=v(x, y), w=(x, y)$ are the displacements along $x, y$ and $z$ axes respectively. The quantity $w^{*}=w^{*}(x, y)$ is an initial imperfection of shell and assumed to be much smaller than thickness $h$ of shell.

The strain components across the shell thickness at a distance $z$ from the mid plane are of the form

$$
\begin{aligned}
& \varepsilon_{x}=\varepsilon_{x}^{0}+z k_{x}, \quad \varepsilon_{y}=\varepsilon_{y}^{0}+z k_{y} \\
& \gamma_{x y}=\gamma_{x y}^{0}+2 z k_{x y} \\
& k_{x}=-w_{, x x}, \quad k_{y}=-w_{, y y}, \quad k_{x y}=-w_{, x y}
\end{aligned}
$$

The stress - strain relationship of cylindrical shell is defined by Hookian law, as

$$
\left(\sigma_{x}, \sigma_{y}\right)=\frac{E}{1-\nu^{2}}\left[\left(\varepsilon_{x}, \varepsilon_{y}\right)+\nu\left(\varepsilon_{y}, \varepsilon_{x}\right)\right], \quad \sigma_{x y}=\frac{E}{2(1+\nu)} \gamma_{x y} .
$$


The force and moment resultants are calculated by

$$
\left\{\left(N_{x}, N_{y}, N_{x y}\right),\left(M_{x}, M_{y}, M_{x y}\right)\right\}=\int_{-h / 2}^{h / 2}\left\{\sigma_{x}, \sigma_{y}, \sigma_{x y}\right\}(1, z) d z .
$$

Substituting Eq. (4) into Eq. (5) and then into Eq. (6), we obtain

$$
\left[\begin{array}{c}
N_{x} \\
N_{y} \\
N_{x y} \\
M_{x} \\
M_{y} \\
M_{x y}
\end{array}\right]=\left[\begin{array}{cccccc}
A_{10} & A_{20} & 0 & A_{11} & A_{21} & 0 \\
A_{20} & A_{10} & 0 & A_{21} & A_{11} & 0 \\
0 & 0 & A_{30} & 0 & 0 & A_{31} \\
A_{11} & A_{21} & 0 & A_{12} & A_{22} & 0 \\
A_{21} & A_{11} & 0 & A_{22} & A_{12} & 0 \\
0 & 0 & A_{31} & 0 & 0 & A_{32}
\end{array}\right]\left[\begin{array}{c}
\varepsilon_{x}^{0} \\
\varepsilon_{y}^{0} \\
\gamma_{x y}^{0} \\
k_{x} \\
k_{y} \\
2 k_{x y}
\end{array}\right]
$$

where the stiffness coefficients $A_{i j} \quad(i=1,2,3, \quad j=0,1,2)$ are calculated by the formulae

$$
\begin{aligned}
A_{1 j} & =\int_{-h / 2}^{h / 2} \frac{E(z)}{1-\nu^{2}(z)} z^{j} d z, \quad A_{2 j}=\int_{-h / 2}^{h / 2} \frac{E(z) \nu(z)}{1-\nu^{2}(z)} z^{j} d z, \\
A_{3 j} & =\int_{-h / 2}^{h / 2} \frac{E(z)}{2[1+\nu(z)]} z^{j} d z=\frac{1}{2}\left(A_{1 j}-A_{2 j}\right) .
\end{aligned}
$$

If the material properties vary in the power law then the coefficients $A_{i j}$ are determined by the analytical expressions in the Appendix. If the material properties vary in the exponential law then the coefficients $A_{i j}$ are determined directly from the formulae (8).

The equilibrium equations of imperfect cylindrical shell are derived from [7]

$$
\begin{gathered}
N_{x, x}+N_{x y, y}=0, \quad N_{x y, x}+N_{y, y}=0 \\
M_{x, x x}+2 M_{x y, x y}+M_{y, y y}+\frac{N_{y}}{R}+N_{x}\left(w_{, x x}+w_{, x x}^{*}\right)+ \\
+2 N_{x y}\left(w_{, x y}+w_{, x y}^{*}\right)+N_{y}\left(w_{, y y}+w_{, y y}^{*}\right)=0 .
\end{gathered}
$$

Assuming the quadratic terms of $w^{*}$ to be omitted, the geometrical compatibility equation deduced from (3) and (4), is

$$
\begin{aligned}
\varepsilon_{x, y y}^{0}+\varepsilon_{y, x x}-\gamma_{x y, x y}^{0}= & \frac{1}{R} w_{, x x}+w_{, x y}^{2}-w_{, x x} w_{, y y}+ \\
& +2 w_{, x y} w_{, x y}^{*}-w_{, x x} w_{, y y}^{*}-w_{, y y} w_{, x x}^{*} .
\end{aligned}
$$

Introducing Airy's stress function $\varphi(x, y)$ so that

$$
N_{x}=\varphi_{, y y}, \quad N_{y}=\varphi_{, x x}, \quad N_{x y}=-\varphi_{, x y} .
$$

It is easy seen that the equations (9) are automatically satisfied. 
The strain components reversely in terms of stress function and deflection are found from Eqs. (7) and (12)

$$
\begin{aligned}
& \varepsilon_{x}^{0}=J_{0}\left(A_{10} \varphi_{, y y}-A_{20} \varphi_{, x x}+J_{1} w_{, x x}+J_{2} w_{, y y}\right), \\
& \varepsilon_{y}^{0}=J_{0}\left(A_{10} \varphi_{, x x}-A_{20} \varphi_{, y y}+J_{1} w_{, y y}+J_{2} w_{, x x}\right), \\
& \gamma_{x y}^{0}=\frac{1}{A_{30}}\left(2 A_{31} w_{, x y}-\varphi_{, x y}\right),
\end{aligned}
$$

where

$$
J_{0}=1 /\left(A_{10}^{2}-A_{20}^{2}\right), \quad J_{1}=A_{10} A_{11}-A_{20} A_{21}, \quad J_{2}=A_{10} A_{21}-A_{20} A_{11} .
$$

Introducing Eq. (13) into Eq. (11) we get

$$
\nabla^{4} \varphi+C_{1} \nabla^{4} w-C_{2}\left[w_{, x y}^{2}-w_{, x x} w_{, y y}-\frac{1}{R} w_{, x x}+2 w_{, x y} w_{, x y}^{*}-w_{, x x} w_{, y y}^{*}-w_{, y y} w_{, x x}^{*}\right]=0
$$

where

$$
C_{1}=J_{2} / A_{10}, \quad C_{2}=1 /\left(A_{10} J_{0}\right) .
$$

To transform Eq. (10), firstly, substituting Eq. (13) into Eq. (7) for finding $M_{i j}$ and then substituting again $M_{i j}$ into Eq. (10), we receive the second governing equation

$$
C_{3} \nabla^{4} \varphi+\frac{1}{R} \varphi_{, x x}+C_{4} \nabla^{4} w+\varphi_{, y y}\left(w_{, x x}+w_{, x x}^{*}\right)-2 \varphi_{, x y}\left(w_{, x y}+w_{, x y}^{*}\right)+\varphi_{, x x}\left(w_{, y y}+w_{, y y}^{*}\right)=0
$$

where

$$
C_{3}=J_{0} J_{2}, \quad C_{4}=J_{0}\left(A_{11} J_{1}+A_{21} J_{2}\right)-A_{12} .
$$

Two equations (15) and (17) are the governing equations used to investigate the nonlinear stability of imperfect FGM cylindrical shells.

\section{Remarks}

If $R \rightarrow \infty$, the equation (15) and (17) become the basic equations to analyze the stability of imperfect FGM plates.

In case $w^{*}=0$, from (15) and (17) we obtain the governing equations for perfect cylindrical shells in [13].

\section{SOLUTION OF THE PROBLEM}

Based on $[13,16]$, the deflection $w$ and initial imperfection $w^{*}$ are chosen in the forms

$$
\begin{aligned}
& w=f\left(\sin \alpha x \sin \beta y+F_{2} \sin ^{2} \alpha x+F_{0}\right), \\
& w^{*}=f_{*}\left(\sin \alpha x \sin \beta y+F_{2} \sin ^{2} \alpha x+F_{0}\right),
\end{aligned}
$$

in which $\alpha=m \pi / L, \beta=n / R$ and $m, n$ are the number of half waves along the $x$ and the number of waves along the $y$ directions respectively. The first term of $w$ (or $w^{*}$ ) in (19) represents a linear buckling shape, and the second term - a nonlinear buckling shape, and the third term - a radial displacement of points belonging to two butt - ends $x=0$ and $x=L$. 
As can be seen that the simply supported boundary condition $w=0, \partial^{2} w / \partial x^{2}=0$ at $x=0, x=L$ is fulfilled on the average sense as

$$
\int_{0}^{2 \pi R} \int_{0}^{L} \frac{\partial w}{\partial x} d x d y=0, \quad \int_{0}^{2 \pi R} \int_{0}^{L} \frac{\partial}{\partial x}\left(\frac{\partial^{2} w}{\partial x^{2}}\right) d x d y=0 .
$$

Substituting of Eq. (19) into Eq. (15) yields

$$
\nabla^{4} \varphi=B_{01} \cos 2 \alpha x+B_{02} \cos 2 \beta y+B_{03} \sin \alpha x \sin \beta y+B_{04} \sin 3 \alpha x \sin \beta y
$$

in which

$$
\begin{aligned}
B_{01} & =\left(8 C_{1} \alpha^{4}-\frac{2}{R} C_{2} \alpha^{2}\right) f F_{2}+\frac{1}{2} C_{2} \alpha^{2} \beta^{2}\left[f^{2}+2 f f_{*}\right], \\
B_{02} & =\frac{1}{2} C_{2} \alpha^{2} \beta^{2}\left[f^{2}+2 f f_{*}\right], \\
B_{03} & =\left[\frac{1}{R} C_{2} \alpha^{2}-C_{1}\left(\alpha^{2}+\beta^{2}\right)^{2}\right] f-C_{2} \alpha^{2} \beta^{2} F_{2}\left[f^{2}+2 f f_{*}\right], \\
B_{04} & =C_{2} \alpha^{2} \beta^{2} F_{2}\left[f^{2}+2 f f_{*}\right] .
\end{aligned}
$$

The general solution of this equation is given by

$$
\begin{aligned}
\varphi=B_{1} \cos 2 \alpha x+B_{2} \cos 2 \beta y & +B_{3} \sin \alpha x \sin \beta y+ \\
& +B_{4} \sin 3 \alpha x \sin \beta y-\frac{1}{2} \sigma_{0 x} h y^{2}-\frac{1}{2} \sigma_{0 y} h x^{2},
\end{aligned}
$$

where $\sigma_{0 x}$ and $\sigma_{0 y}$ are the negative average longitudinal stress and circumferential stress respectively, and

$$
B_{1}=\frac{B_{01}}{16 \alpha^{4}}, \quad B_{2}=\frac{B_{02}}{16 \beta^{4}}, \quad B_{3}=\frac{B_{03}}{\left(\alpha^{2}+\beta^{2}\right)^{2}}, \quad B_{4}=\frac{B_{04}}{\left(9 \alpha^{2}+\beta^{2}\right)^{2}},
$$

or

$$
\begin{array}{ll}
B_{1}=a_{1} F_{2} f+a_{2}\left(f^{2}+2 f f_{*}\right), & B_{2}=a_{3}\left(f^{2}+2 f f_{*}\right), \\
B_{3}=a_{4} F_{2}\left(f^{2}+2 f f_{*}\right)+a_{5} f, & B_{4}=a_{6} F_{2}\left(f^{2}+2 f f_{*}\right),
\end{array}
$$

in which

$$
\begin{aligned}
& a_{1}=\frac{1}{8}\left(4 C_{1}-\frac{C_{2}}{R \alpha^{2}}\right), \quad a_{2}=\frac{C_{2} \beta^{2}}{32 \alpha^{2}}, \quad a_{3}=\frac{C_{2} \alpha^{2}}{32 \beta^{2}}, \\
& a_{4}=-\frac{C_{2} \alpha^{2} \beta^{2}}{\left(\alpha^{2}+\beta^{2}\right)^{2}}, \quad a_{5}=\frac{C_{2} \alpha^{2}}{R\left(\alpha^{2}+\beta^{2}\right)^{2}}-C_{1}, \quad a_{6}=\frac{C_{2} \alpha^{2} \beta^{2}}{\left(9 \alpha^{2}+\beta^{2}\right)^{2}} .
\end{aligned}
$$

In order to establish a load - deflection curve, first of all introducing $w, w_{*}$ and $\varphi$ into the left side of Eq. (17) denoted by $\phi_{1}$ then applying Galerkin's method

$$
\int_{0}^{2 \pi R} \int_{0}^{L} \phi_{1} d x d y=0, \quad \int_{0}^{2 \pi R} \int_{0}^{L} \phi_{1} \sin \alpha x \sin \beta x d x d y=0, \quad \int_{0}^{2 \pi R} \int_{0}^{L} \phi_{1} \sin ^{2} \alpha x d x d y=0
$$

lead to

$$
\sigma_{0 y}=0
$$




$$
\begin{gathered}
\left\{\left[\left(\alpha^{2}+\beta^{2}\right)^{2} C_{3}-\frac{1}{R} \alpha^{2}\right]\left[a_{4} F_{2} f\left(f+2 f_{*}\right)+a_{5} f\right]+C_{4} f\left(\alpha^{2}+\beta^{2}\right)^{2}\right\}-\left(f+f_{*}\right) \alpha^{2} \beta^{2} . \\
\cdot\left\{2\left[a_{1} F_{2} f+\left(a_{2}+a_{3}\right) f\left(f+2 f_{*}\right)\right]+F_{2}\left[\left(a_{6}-a_{4}\right) F_{2} f\left(f+2 f_{*}\right)-a_{5} f\right]-h\left(\frac{\sigma_{0 x}}{\beta^{2}}+\frac{\sigma_{0 y}}{\alpha^{2}}\right)\right\}=0 \\
\frac{2}{R} \sigma_{0 y} h=\left\{\left(16 \alpha^{4} C_{3}-\frac{4}{R} \alpha^{2}\right) a_{2} f\left(f+2 f_{*}\right)+F_{2} f\left[a_{1}\left(16 \alpha^{4} C_{3}-\frac{4}{R} \alpha^{2}\right)-8 \alpha^{4} C_{4}\right]\right\}+ \\
+\left(f+f_{*}\right) \alpha^{2} \beta^{2}\left\{\left[\left(a_{6}-a_{4}\right) F_{2} f\left(f+2 f_{*}\right)-a_{5} f\right]-2 \sigma_{0 x} h F_{2} / \beta^{2}\right\} .
\end{gathered}
$$

In addition to three equations $(24,25,26)$ the cylindrical shell must also satisfy the circumferential closed condition $[13,16]$ as

$$
\int_{0}^{2 \pi R} \int_{0}^{L} v_{, y} d x d y=0
$$

Using Eqs. (3) and (13), this integral becomes

$$
\int_{0}^{2 \pi R} \int_{0}^{L}\left\{J_{0}\left(A_{10} \varphi_{, x x}-A_{20} \varphi_{, y y}+J_{2} w_{, x x}+J_{1} w_{, y y}\right)+\frac{w}{R}-\frac{1}{2} w_{, y}^{2}-w_{, y} w_{, y}^{*}\right\} d x d y=0 .
$$

After some calculations we get

$$
\sigma_{0 y}=\frac{1}{A_{10} h}\left[\frac{2 f F_{0}+f F_{2}}{2 J_{0} R}-\frac{\beta^{2}}{8 J_{0}}\left(f^{2}+2 f f^{*}\right)+A_{20} \sigma_{0 x} h\right] .
$$

Because of condition (24), Eqs. (25), (26) and (27) lead to

$$
\begin{gathered}
\left\{\left[\left(\alpha^{2}+\beta^{2}\right)^{2} C_{3}-\frac{1}{R} \alpha^{2}\right]\left[a_{4} F_{2} f\left(f+2 f_{*}\right)+a_{5} f\right]+C_{4} f\left(\alpha^{2}+\beta^{2}\right)^{2}\right\}-\left(f+f_{*}\right) \alpha^{2} \beta^{2} . \\
\cdot\left\{2\left[a_{1} F_{2} f+\left(a_{2}+a_{3}\right) f\left(f+2 f_{*}\right)\right]+F_{2}\left[\left(a_{6}-a_{4}\right) F_{2} f\left(f+2 f_{*}\right)-a_{5} f\right]-h \frac{\sigma_{0 x}}{\beta^{2}}\right\}=0 . \\
\left\{\left(16 \alpha^{4} C_{3}-\frac{4}{R} \alpha^{2}\right) a_{2} f\left(f+2 f_{*}\right)+F_{2} f\left[a_{1}\left(16 \alpha^{4} C_{3}-\frac{4}{R} \alpha^{2}\right)-8 \alpha^{4} C_{4}\right]\right\}+ \\
+\left(f+f_{*}\right) \alpha^{2} \beta^{2}\left\{\left[\left(a_{6}-a_{4}\right) F_{2} f\left(f+2 f_{*}\right)-a_{5} f\right]-2 \sigma_{0 x} h F_{2} / \beta^{2}\right\}=0 . \\
{\left[\frac{2 f F_{0}+f F_{2}}{2 J_{0} R}-\frac{\beta^{2}}{8 J_{0}}\left(f^{2}+2 f f^{*}\right)+A_{20} \sigma_{0 x} h\right]=0 .}
\end{gathered}
$$

Two equations (28) and (29) are the governing equations used to find the critical buckling load and analyse the postbuckling load - deflection curves for imperfect FGM cylindrical shells under axial compression. The equation (30) is used to determine the coefficient $F_{0}$. The equations (28) and (29) will have a more convenient form if the following nondimensional parameters are introduced

$$
\xi_{1}=\frac{f}{h}, \quad \xi_{2}=\frac{f F_{2}}{h}, \quad \xi_{0}=\frac{f F_{0}}{h}, \quad \xi_{*}=\frac{f_{*}}{h} \Rightarrow F_{2}=\frac{\xi_{2}}{\xi_{1}} .
$$


Then Eq. (28) and Eq. (29) are rewritten in the form

$$
\begin{array}{r}
\sigma_{0 x}=\beta_{*}^{2}\left\{2\left[a_{1}^{*} \xi_{2}+\left(a_{2}^{*}+a_{3}^{*}\right) \xi_{1}\left(\xi_{1}+2 \xi_{*}\right)\right]+\left[\left(a_{6}^{*}-a_{4}^{*}\right) \xi_{2}\left(\xi_{2}+2 \xi_{*} \frac{\xi_{2}}{\xi_{1}}\right)-a_{5}^{*} \xi_{2}\right]\right\} \frac{h^{2}}{R^{2}} \\
-\frac{1}{\alpha_{*}^{2}\left(\xi_{1}+\xi_{*}\right)}\left\{\left[\left(\alpha_{*}^{2}+\beta_{*}^{2} \frac{L^{2}}{R^{2}}\right)^{2} C_{3}^{*} \frac{h^{2}}{L^{2}}-\alpha_{*}^{2} \frac{h}{R}\right] \cdot\left[a_{4}^{*} \xi_{2}\left(\xi_{1}+2 \xi_{*}\right)+a_{5}^{*} \xi_{1}\right]+\right. \\
\sigma_{0 x}=\frac{\beta_{*}^{2} \xi_{1}}{2 \xi_{2}}\left[\left(a_{*}^{2}+\beta_{*}^{2} \frac{L^{2}}{R^{2}}\right)^{2} \frac{h^{2}}{L^{2}}\right\}, \\
\cdot\left\{\left(16 \alpha_{*}^{4} C_{3}^{*} \frac{h^{2}}{L^{2}}-4 \alpha_{*}^{2} \frac{h}{R}\right) \xi_{2}\left(\xi_{2}+2 \xi_{*}\right)-a_{5}^{*} \xi_{1}\right] \frac{h^{2}}{R^{2}}+\frac{\xi_{1}}{2 \xi_{2}\left(\xi_{1}+\xi_{*}\right) \alpha_{*}^{2}} .
\end{array}
$$

in which

$$
\begin{aligned}
& A_{10}^{*}=A_{10} / h, \quad A_{20}^{*}=A_{20} / h, \quad A_{30}^{*}=A_{30} / h, \\
& A_{11}^{*}=A_{11} / h^{2}, \quad A_{21}^{*}=A_{21} / h^{2}, \quad A_{31}^{*}=A_{31} / h^{2}, \\
& A_{12}^{*}=A_{12} / h^{3}, \quad A_{22}^{*}=A_{22} / h^{3}, A_{32}^{*}=A_{32} / h^{3} . \\
& E_{1}^{*}=E_{1} / h, \quad E_{2}^{*}=E_{2} / h^{2}, \quad E_{3}^{*}=E_{3} / h^{3}, \quad D^{*}=D / h^{3} . \\
& J_{0}^{*}=J_{0} h^{2}=1 /\left(A_{10}^{* 2}-A_{20}^{* 2}\right), \quad J_{1}^{*}=J_{1} / h^{3}=\left(A_{10}^{*} A_{11}^{*}-A_{20}^{*} A_{21}^{*}\right), \\
& J_{2}^{*}=J_{2} / h^{3}=\left(A_{10}^{*} A_{21}^{*}-A_{20}^{*} A_{11}^{*}\right) . \\
& C_{1}^{*}=C_{1} / h^{2}=J_{2}^{*} / A_{10}^{*}, \quad C_{2}^{*}=C_{2} / h=1 /\left(A_{10}^{*} J_{0}^{*}\right), \\
& C_{3}^{*}=C_{3} / h=J_{0}^{*} J_{2}^{*}, \quad C_{4}^{*}=C_{4} / h^{3}=J_{0}^{*}\left(A_{11}^{*} J_{1}^{*}+A_{21}^{*} J_{2}^{*}\right)-A_{12}^{*} . \\
& \alpha^{*}=\alpha L=m \pi, \quad \beta^{*}=\beta R=n . \\
& a_{1}^{*}=\frac{a_{1}}{h^{2}}=\frac{1}{8}\left(4 C_{1}^{*}-\frac{C_{2}^{*}}{\alpha_{*}^{2}} \frac{h}{R} \frac{L^{2}}{h^{2}}\right), \quad a_{2}^{*}=\frac{a_{2}}{h}=\frac{C_{2}^{*} \beta_{*}^{2}}{32 \alpha_{*}^{2}} \cdot \frac{L^{2}}{R^{2}}, \\
& a_{3}^{*}=\frac{a_{3}}{h}=\frac{C_{2}^{*} \alpha_{*}^{2}}{32 \beta_{*}^{2}} \cdot \frac{R^{2}}{L^{2}}, \quad a_{4}^{*}=\frac{a_{4}}{h}=-\frac{C_{*}^{2} \beta_{*}^{2}}{\left[\alpha_{*}^{2}+\beta_{*}^{2}(L / R)^{2}\right]^{2}} \frac{L^{2}}{R^{2}}, \\
& a_{5}^{*}=\frac{a_{5}}{h^{2}}=\frac{C_{2}^{*} \alpha_{*}^{2}}{\left[\alpha_{*}^{2}+\beta_{*}^{2}(L / R)^{2}\right]^{2}} \frac{L^{2}}{R h}-C_{1}^{*}, \quad a_{6}^{*}=\frac{a_{6}}{h}=\frac{C_{2}^{*} \alpha_{*}^{2} \beta_{*}^{2}}{\left[9 \alpha_{*}^{2}+\beta_{*}^{2}(L / R)^{2}\right]^{2}} \frac{L^{2}}{R^{2}} .
\end{aligned}
$$

\section{PERFECT CYLINDRICAL SHELLS}

In this case $f_{*}=0$, Eq. (28) is written as

$$
\begin{aligned}
& \left\{\left[\left(\alpha^{2}+\beta^{2}\right)^{2} C_{3}-\frac{1}{R} \alpha^{2}\right]\left[a_{4} F_{2} f^{2}+a_{5} f\right]+C_{4} f\left(\alpha^{2}+\beta^{2}\right)^{2}\right\}- \\
& -f \alpha^{2} \beta^{2}\left\{2\left[a_{1} F_{2} f+\left(a_{2}+a_{3}\right) f^{2}\right]+F_{2}\left[\left(a_{6}-a_{4}\right) F_{2} f^{2}-a_{5} f\right]-\frac{h \sigma_{0 x}}{\beta^{2}}\right\}=0 .
\end{aligned}
$$


Putting $f_{2}=F_{2} f$ and solving $f^{2}$, we obtain

$$
f^{2}=-\frac{H_{01}+H_{04} f_{2}^{2}+H_{05} f_{2}-\frac{\alpha^{2}}{2} h \sigma_{0 x}}{H_{03}}
$$

in which

$$
\begin{aligned}
& H_{01}=-\frac{1}{2}\left\{\left[\left(\alpha^{2}+\beta^{2}\right)^{2} C_{3}-\frac{1}{R} \alpha^{2}\right] a_{5}+C_{4}\left(\alpha^{2}+\beta^{2}\right)^{2}\right\}, \\
& H_{03}=\alpha^{2} \beta^{2}\left(a_{2}+a_{3}\right), \quad H_{04}=\frac{\alpha^{2} \beta^{2}}{2}\left(a_{6}-a_{4}\right), \\
& H_{05}=-\frac{1}{2}\left\{\left[\left(\alpha^{2}+\beta^{2}\right)^{2} C_{3}-\frac{1}{R} \alpha^{2}\right] a_{4}+\alpha^{2} \beta^{2}\left(a_{5}-2 a_{1}\right)\right\} .
\end{aligned}
$$

For Eq. (29), also taking $f_{*}=0$, leads to

$$
\begin{aligned}
\left(8 \alpha^{4} C_{3}-\frac{2}{R} \alpha^{2}\right) a_{2} f^{2} & +F_{2} f\left[a_{1}\left(8 \alpha^{4} C_{3}-\frac{2}{R} \alpha^{2}\right)-4 \alpha^{4} C_{4}\right]+ \\
& +\frac{\alpha^{2} \beta^{2}}{2}\left[\left(a_{6}-a_{4}\right) F_{2} f^{3}-a_{5} f^{2}\right]-\alpha^{2} \sigma_{0 x} h F_{2} f=0 .
\end{aligned}
$$

One gets

$$
\sigma_{0 x} h=\left[H_{06}+f^{2}\left(H_{08}+H_{07} / f_{2}\right)\right] / \alpha^{2}
$$

where

$$
\begin{gathered}
H_{06}=a_{1}\left(8 \alpha^{4} C_{3}-\frac{2}{R} \alpha^{2}\right)-4 \alpha^{4} C_{4}, \quad H_{07}=\left(8 \alpha^{4} C_{3}-\frac{2}{R} \alpha^{2}\right) a_{2}-\frac{\alpha^{2} \beta^{2}}{2} a_{5}, \\
H_{08}=\frac{\alpha^{2} \beta^{2}}{2}\left(a_{6}-a_{4}\right) .
\end{gathered}
$$

Substituting $f^{2}$ from Eq. (34) into Eq. (36) gives us

$$
\begin{gathered}
\sigma_{0 x}=\frac{2}{\left[H_{07}+\left(H_{08}-2 H_{03}\right) f_{2}\right] h \alpha^{2}}\left[H_{01} H_{07}+\left(H_{01} H_{08}+H_{05} H_{07}-H_{03} H_{06}\right) f_{2}+\right. \\
\left.+\left(H_{04} H_{07}+H_{05} H_{08}\right) f_{2}^{2}+H_{04} H_{08} f_{2}^{3}\right] .
\end{gathered}
$$

This is the relation found in the paper [13]. The equation (38) is used to investigate load - deflection curves. It is also used to find buckling critical loads from the condition $d \sigma_{0 x} / d f_{2}=0$.

Now, consider the casec $f_{2}=0$, i.e. the nonlinear buckling shape is ignored, Eq. (38) becomes

$$
\sigma_{0 x}=\frac{2 H_{01}}{h \alpha^{2}} .
$$

Minimizing this expression, leads to

$$
\sigma_{x c l}=\frac{C_{2}}{h R^{2}} \sqrt{\frac{R^{2}\left(C_{1} C_{3}-C_{4}\right)}{C_{2}}}-\frac{1}{R h}\left(C_{2} C_{3}+C_{1}\right)-\frac{C_{4}-C_{1} C_{3}}{h} \sqrt{\frac{C_{2}}{R^{2}\left(C_{1} C_{3}-C_{4}\right)}} .
$$


Substituting from (14) and (16)

$$
\begin{aligned}
& C_{1}=\frac{A_{10} A_{21}-A_{20} A_{11}}{A_{10}}, \quad C_{2}=\frac{A_{10}^{2}-A_{20}^{2}}{A_{10}}, \quad C_{3}=\frac{A_{10} A_{21}-A_{20} A_{11}}{A_{10}^{2}-A_{20}^{2}}, \\
& C_{4}=\frac{1}{A_{10}^{2}-A_{20}^{2}}\left[A_{11}\left(A_{10} A_{11}-A_{20} A_{21}\right)+A_{21}\left(A_{10} A_{21}-A_{20} A_{11}\right)\right]-A_{12}
\end{aligned}
$$

into the above expression, after some calculations, the critical load of perfect FG cylindrical shells with the linear buckling shape is obtained as follows

$$
\sigma_{x c l}=\frac{2}{h R A_{10}}\left[\sqrt{\left(A_{10}^{2}-A_{20}^{2}\right)\left(A_{12} A_{10}-A_{11}^{2}\right)}-\left(A_{10} A_{21}-A_{20} A_{11}\right)\right] .
$$

This result coincides with the one of [13].

Thus, from general results (28) and (29) for imperfect FG cylindrical shells we can return to the results published in the paper [13] for perfect FG cylindrical shells.

\section{ISOTROPIC PERFECT CYLINDRICAL SHELL}

In this case, we have $C_{1}=C_{3}=0, \quad C_{2}=E h, \quad C_{4}=-E h^{3} /\left[12\left(1-\nu^{2}\right)\right]$.

One gets

$$
A_{10} A_{21}-A_{20} A_{11}=0, \quad A_{10}^{2}-A_{20}^{2}=A_{10} E h, \quad A_{11}^{2}-A_{10} A_{12}=-A_{10} E h^{3} /\left[12\left(1-\nu^{2}\right)\right] .
$$

Introdution of these coefficients into Eq. (40) gives us

$$
\sigma_{c l \min }=\frac{E h}{R \sqrt{3\left(1-\nu^{2}\right)}}
$$

This is the minimum value of axial compressive load found in [16] based on the classical shell theory.

\section{NUMERICAL CALCULATIONS AND DISCUSSIONS}

Problem 1: Comparision with the results of Huang and Han [13].

Consider a FGM cylindrical shell is made from two materials Zirconia and Ti-6Al-4V which take the values as follows [13].

Table 1. Temperature coefficients for the material properties of Zirconia and $\mathrm{Ti}-6 \mathrm{Al}-4 \mathrm{~V}$

\begin{tabular}{|c|c|c|c|c|c|}
\hline Material properties & $\mathrm{c}_{0}$ & $\mathrm{c}_{-1}$ & $\mathrm{c}_{1}$ & $\mathrm{c}_{2}$ & $\mathrm{c}_{3}$ \\
\hline \multicolumn{7}{|c|}{ Zirconia } \\
\hline $\mathrm{E}_{C}(\mathrm{~Pa})$ & $244.26596^{*} 10^{9}$ & 0 & $-1.3707^{*} 10^{-3}$ & $1.21393^{*} 10^{-6}$ & $-3.681378^{*} 10^{-10}$ \\
\hline$\nu_{C}$ & 0.2882 & 0 & $1.13345^{*} 10^{-4}$ & 0 & 0 \\
\hline \multicolumn{7}{|c|}{ Ti-6Al-4V } \\
\hline $\mathrm{E}_{m}(\mathrm{~Pa})$ & $122.55676^{*} 10^{9}$ & 0 & $-4.58535^{*} 10^{-4}$ & 0 & 0 \\
\hline$\nu_{m}$ & 0.28838235 & 0 & $1.12136^{*} 10^{-4}$ & 0 & 0 \\
\hline
\end{tabular}


According to [13] the material properties depend on temperature $\mathrm{T}$ as the following

$$
P_{m}(T)=c_{0}\left(c_{-1} T^{-1}+1+c_{1} T+c_{2} T^{2}+c_{3} T^{3}\right) .
$$

In this paper, we consider $\mathrm{T}=300 \mathrm{~K}$. For other temperature field, one can do that by the same method based on the formula (42).



Fig. 2. Effects of buckling mode ( $m=1, n$ changing) on $\sigma_{0 x}-f_{2}$ curves

With $k=k_{1}=1, \mathrm{~T}=300 \mathrm{~K}, \mathrm{R} / \mathrm{h}=200, \mathrm{~L} / \mathrm{R}=1, f_{*}=0$ according to the formula (38) and programming Matlab software we receive results given in Fig. 2 and Fig. 3 . These



Fig. 3. Effects of buckling mode on $\sigma_{0 x}-f_{2}$ curves

results describe the relationship $\sigma_{0 x}-f_{2}$ when the buckling mode is changed. As can be 
observed, the smallest value $\sigma_{0 x c r}=79.0409 \mathrm{MPa}$ corresponds to $(m, n)=(1,6)$. This result is in very good agreement with the one of Huang $\left(\sigma_{c r}=79.10 \mathrm{MPa}\right.$ with buckling mode $(m, n)=(1,6))$ obtained by other method. This result also has affirmed the accuracy of proposed method.

Problem 2: Effects of geometric parameters.

Based on the formula (38), with $\mathrm{T}=300 \mathrm{~K}, k=k_{1}=1, f_{*}=0$, consider two cases:

- The ratio $R / h$ is varied from 200 to 1000 , while $R / L=1$.

- The ratio $R / L$ takes values from 0.5 to 4 , while $R / h=200$.

Critical loads (MPa) are given in the Table 2 and Table 3, respectively.

Table 2. Effects of geometric parameter $R / h$ to critical load

\begin{tabular}{|c|c|c|}
\hline \multirow{2}{*}{$R / h$} & \multicolumn{2}{|c|}{$L / R=1$} \\
\cline { 2 - 3 } & Power law & Exponential law \\
\hline 200 & $79.0409(1,6)^{*}$ & $78.4455(1,6)$ \\
\hline 300 & $53.0174(1,6)$ & $52.5480(1,6)$ \\
\hline 400 & $40.3310(1,7)$ & $39.9932(1,7)$ \\
\hline 500 & $33.5713(1,7)$ & $33.2663(1,7)$ \\
\hline 600 & $27.3734(2,11)$ & $27.1678(2,11)$ \\
\hline 700 & $23.0498(2,11)$ & $22.8651(2,11)$ \\
\hline 800 & $19.7602(2,12)$ & $19.6114(2,12)$ \\
\hline 900 & $17.3047(2,12)$ & $17.1678(2,12)$ \\
\hline 1000 & $15.5465(2,12)$ & $15.4181(2,12)$ \\
\hline
\end{tabular}

(* The numbers in the parenthesis denote the buckling modes $(m, n))$.

As shown in the Table 2 , the more the value of ratio $R / h$ is big, the more the value of critical load is small. This result agrees with the real property of struture i.e. the shell is thinner the value of critical load is smaller.

Table 3. Effects of geometrical parameter $L / R$ to critical load

\begin{tabular}{|c|c|c|}
\hline \multirow{2}{*}{$L / R$} & \multicolumn{2}{|c|}{$R / h=200$} \\
\cline { 2 - 3 } & Power law & Exponential law \\
\hline 0.5 & $116.8459(1,9)$ & $116.0977(1,9)$ \\
\hline 1 & $79.0409(1,6)$ & $78.4455(1,6)$ \\
\hline 1.5 & $84.4439(1,5)$ & $83.7531(1,5)$ \\
\hline 2 & $79.0409(2,6)$ & $78.4455(2,6)$ \\
\hline 4 & $78.8411(3,5)$ & $78.1775(3,5)$ \\
\hline
\end{tabular}

As can be seen from Table 3 when the ratio $L / R$ increases from 0.5 to 1 , the critical load is decreases very much, whereas when $L / R$ varies from 1 to 4 , the critical load is changed less. That means the nonlinear response of short cylindrical shell is very sensitive to a variation of ratio $L / R$.

Table 2 and Table 3 also show that, in this example, when material properties of shell obey the power and exponential laws, both their critical loads are nearly equal. 
Problem 3: Relation between critical load and buckling modes.

Consider the case $k=k_{1}=1, \mathrm{~T}=300 \mathrm{~K}, R / h=200, L / R=1, f^{*}=0$. Couple $(m, n)$ receives the values of from 1 to 10 and based on the expression (38) we establish the relationship table between the critical load $\sigma_{0 x}(\mathrm{MPa})$ and buckling modes $(m, n)$.

First of all, we find the relationship between critical load and buckling mode $(m, n)$ when material properties accord with the power laws. Using matlab software we received $\sigma_{0 x c r}=79.0409 \mathrm{MPa}$ corresponding to $m=1, n=6$. This result coincides with the results received in the Problem 1.

By the same method, the numerical results concerning the relationship between critical load and buckling mode when material properties are according to the exponential law, are given by Table 4 .

Table 4. Relationship between critical load and buckling mode $(m, n)$ when the material properties according to the exponential law

\begin{tabular}{|c|c|c|c|c|c|c|c|c|c|c|}
\hline$(m, n)$ & $n=1$ & 2 & 3 & 4 & 5 & 6 & 7 & 8 & 9 & 10 \\
\hline$m=1$ & 3232.500 & 1812.600 & 632.8914 & 207.7360 & 94.6486 & 78.4455 & 95.7017 & 131.3731 & 182.8654 & 248.7592 \\
\hline 2 & 901.5650 & 851.6488 & 698.8532 & 489.7799 & 313.9233 & 201.3150 & 141.6480 & 117.5431 & 116.0952 & 129.7078 \\
\hline 3 & 489.2352 & 483.6904 & 462.1146 & 416.5820 & 353.1614 & 287.8135 & 233.3697 & 194.9493 & 172.4518 & 163.6833 \\
\hline 4 & 407.4831 & 406.0168 & 399.9857 & 385.5725 & 361.1792 & 329.5046 & 296.1243 & 266.3040 & 243.2913 & 228.4006 \\
\hline 5 & 439.1634 & 438.3880 & 435.1254 & 426.9829 & 412.2492 & 391.4036 & 367.1844 & 343.1002 & 322.0465 & 305.8588 \\
\hline 6 & 527.9270 & 520.9976 & 496.5251 & 481.6082 & 459.2220 & 446.9971 & 435.0867 & 420.0367 & 402.6932 & 387.7014 \\
\hline 7 & 549.5604 & 476.5911 & 449.4931 & 436.0079 & 427.2743 & 420.7412 & 415.6931 & 412.0529 & 409.9949 & 409.7943 \\
\hline 8 & 679.8904 & 540.8819 & 480.0267 & 452.0387 & 437.6079 & 429.6960 & 425.5425 & 424.0116 & 424.6567 & 427.3523 \\
\hline 9 & 868.0580 & 673.8740 & 567.7404 & 514.2321 & 486.0402 & 470.7716 & 462.8584 & 459.6660 & 459.8931 & 462.8902 \\
\hline 10 & 1097.100 & 867.0790 & 712.3330 & 624.0947 & 573.9992 & 545.0943 & 528.6159 & 520.0056 & 516.8099 & 517.6652 \\
\hline
\end{tabular}

From Table 4, we see that in both cases the buckling load is the smallest one when $(m, n)=(1,6)$. This result also is very near to the result of Problem 1 .

Problem 4: Comparison between critical loads of nonlinear buckling shape and linear shape.

In this part, numerical critical load results of two buckling shapes are presented by using Eq. (38) and Eq. (40) with the parameters $\mathrm{T}=300 \mathrm{~K}, k=k 1=1, f^{*}=0$ (see Table $5)$.

Table 5. Critical load (MPa) of two buckling shapes

\begin{tabular}{|c|c|c|}
\hline \multirow{2}{*}{$R / h$} & \multicolumn{2}{|c|}{$L / R=1$} \\
\cline { 2 - 3 } & \multicolumn{2}{|c|}{ Power law } \\
\cline { 2 - 3 } & Nonlinear buckling shape calculated by Eq. (38) & Linear buckling shape calculated by Eq. (40) \\
\hline 200 & $79.0409(1,6)$ & $410.3914(1,6)$ \\
\hline 300 & $53.0174(1,6)$ & $273.5943(1,6)$ \\
\hline 400 & $40.3310(1,7)$ & $205.1957(1,7)$ \\
\hline 500 & $33.5713(1,7)$ & $164.1566(1,7)$ \\
\hline 600 & $27.3734(2,11)$ & $136.7971(2,11)$ \\
\hline 700 & $23.0498(2,11)$ & $117.2547(2,11)$ \\
\hline 800 & $19.7602(2,12)$ & $102.5979(2,12)$ \\
\hline 900 & $17.3047(2,12)$ & $91.1981(2,12)$ \\
\hline 1000 & $15.5465(2,12)$ & $82.0783(2,12)$ \\
\hline
\end{tabular}


As shown in Table 5, the critical load with nonlinear buckling shape is smaller than the one with linear buckling shape.

Problem 5: Effects of imperfection.

Now we use Eqs. (32) and (33) to plot curves describing $\sigma_{0 x}-\xi_{2}$ relationship for imperfect cylindrical shells.

In each figure below (Figs. 4, 5, 6, 7, 8), two graphs are given: Graph in Fig.(a) with the small subdivided scale on the axis of abscissae $\xi_{2}$ to indicate clearly the starting point of the $\sigma_{0 x}-\xi_{2}$ curve at the origin $\mathrm{O}$, while in Fig.(b) with the larger scale of abscissae to indicate extremum type buckling of imperfect FGM shells.



(a)

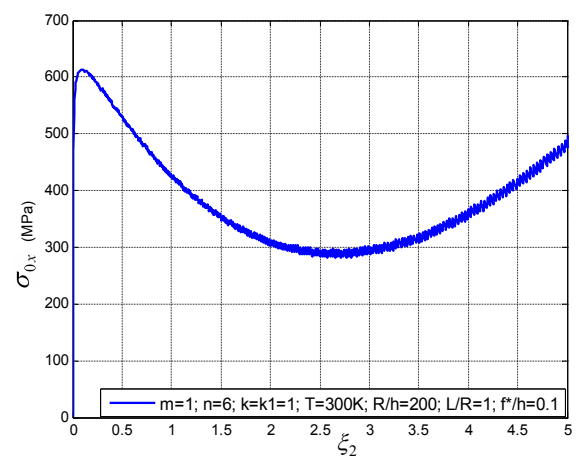

(b)

Fig. 4. Curve describeing the relationship $\sigma_{0 x}-\xi_{2}$ of imperfect FG cylindrical shells

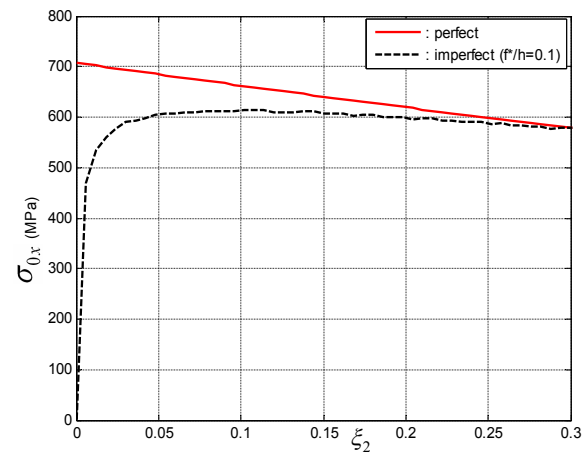

(a)

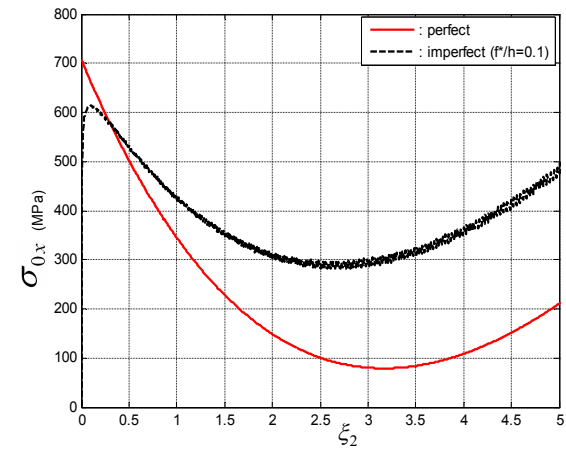

(b)

Fig. 5. Curves describeing the relationship $\sigma_{0 x}-\xi_{2}$ of perfect and imperfect FG cylindrical shells $\left(m=1, n=6, k=k_{1}=1, \mathrm{~T}=300 \mathrm{~K}, R / h=200, L / R=1\right)$

The Fig. 4 and Fig. 5 show, for imperfect cylindrical shells, when an axial compressive load starts different to zero then immediately is appeared a buckling (i.e. a deflection 




(a)



(b)

Fig. 6. Effects of volume fraction indexes $\left(m=1, n=6, \xi_{*}=0.1, \mathrm{~T}=300 \mathrm{~K}\right.$, $R / h=200, L / R=1)$

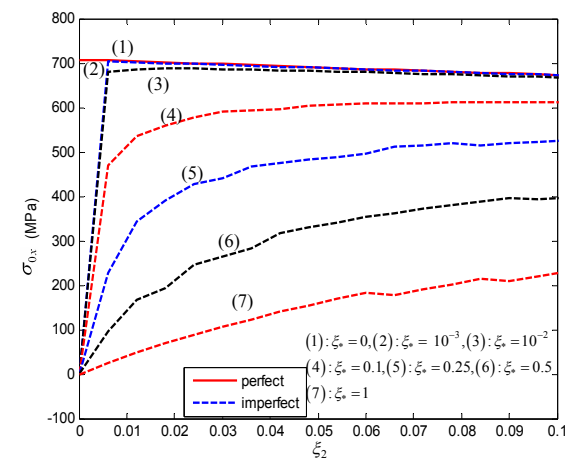

(a)

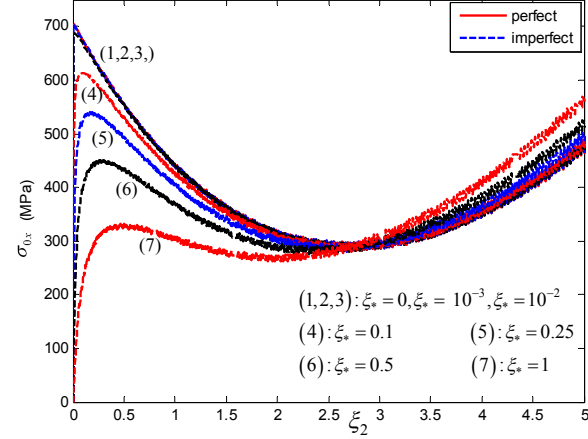

(b)

Fig. 7. Effect of imperfection $\left(m=1, n=6, k=k_{1}=1, \mathrm{~T}=300 \mathrm{~K}, R / h=200\right.$, $L / R=1)$

is different to zero), while with perfect cylindrical shells only is appeared a buckling when compressive load reaches a certain critical value. Furthermore, during the first stage, the $\sigma_{0 x}-\xi_{2}$ curve is close to the axis of ordinates $\sigma_{0 x}$, the buckling occurs slowly until the load reaches an upper critical value. In the second stage, although a load decreases but a buckling still occurs. At these both stages the critical load of imperfect FG cylindrical shell is smaller than the critical load of the perfect FG shell. To the third stage, when $\xi_{2}$ exceeds a special value, an inverse trend occurs. This similar phenomenon is also reflected in the paper [11] when the authors studied the nonlinear stability of imperfect FGM cylindrical panels.

The effects of volume fraction indexes $\mathrm{k}$ and $k_{1}$ on $\sigma_{0 x}-\xi_{2}$ curves are illustrated in Fig. 6. Graph is plotted with $k=k_{1}=0,1,5$ for perfect and imperfect cylindrical shells. 


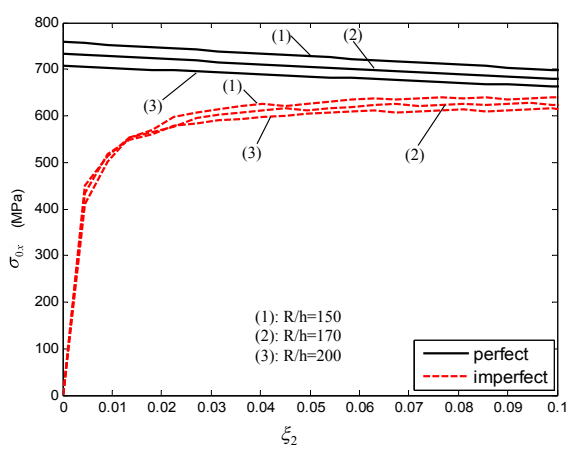

(a)

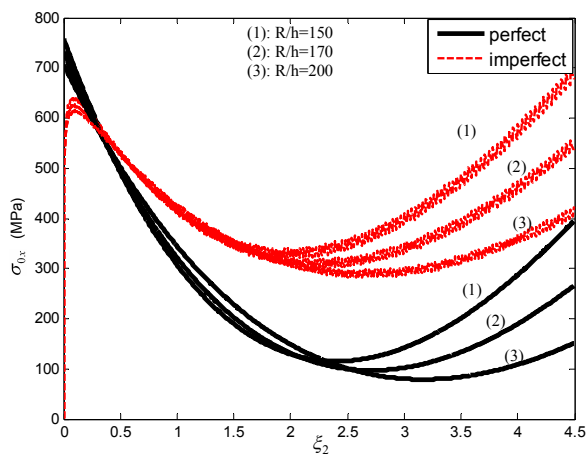

(b)

Fig. 8. Effects of the ratio $R / h\left(m=1, n=6, k=k_{1}=1, \mathrm{~T}=300 \mathrm{~K}, \xi_{*}=0.1\right.$, $L / R=1)$

As can be observed in both cases, the more the indixes $k=k_{1}$ increase the more the $\sigma_{0 x}-\xi_{2}$ curve is lowered.

The effects of imperfection on $\sigma_{0 x}-\xi_{2}$ curves are given in Fig. 7. The graph is plotted with $\xi_{*}=0, \xi_{*}=0.001, \xi_{*}=0.01, \xi_{*}=0.1, \xi_{*}=0.25, \xi_{*}=0.5, \xi_{*}=1$. It is easy to see the more the imperfection $\xi_{*}$ is large the more the $\sigma_{0 x}-\xi_{2}$ curve is sloped i.e. the shells become more unstable.

The effect of geometric ratio $R / h$ on $\sigma_{0 x}-\xi_{2}$ curve of perfect and imperfect shells are reflected in Fig. 8. As can be seen, the critical load bearing capacity of shells decreases when the $R / h$ ratio increases.

\section{CONCLUSIONS}

In this paper the results obtained by Huang and Han in [13] have been developed for imperfect functionally graded cylindrical shells using Galerkin's method. The expanded results return to the ones of [13] in the case of perfect cylindrical shells. The power and exponential laws for material property variation are utilized to find expression of critical loads for imperfect and perfect cylindrical shells and postbuckling load - deflection curves. Effects of buckling modes of fraction volume indexes, initial imperfection and geometric parameters on the critical loads and postbuckling load - deflection curves are presented. Comparisons between the linear and nonlinear buckling shape are given.

\section{ACKNOWLEDGEMENT}

The authors would like to express their sincere thank to Professor Dao Huy Bich for offering valuable suggestions to this paper completed.

This paper was supported by the National Foundation for Science and Technology Development of Vietnam - NAFOSTED. The authors are grateful for this financial support. 


\section{REFERENCES}

[1] Koizumi M., FGM activities Japan, Composite Part B: Engineering, 28 (1997) 1 - 4.

[2] Shen H. S., Postbuckling analysis of axially-loaded functionally graded cylindrical shells in thermal environments, Compos Sci and Tech, 62 (2002) 977 - 987.

[3] Shen H.S, Noda N., Postbuckling of FGM cylindrical shells under combined axial and radial mechanical loads in thermal environments, Int J Solids and Struct, 42 (2005) 4641 - 4662.

[4] Shahsiah R., Eslami M. R., Functionally graded cylindrical shell thermal instability base on improved Donnell equations, AIAA J, 41 (2003) 1819 - 1826.

[5] Wu L., Jiang Z., Lin J., Thermoelastic stability of functionally graded cylindrical shells, Compos Struct, 70 (2005) 60 - 68.

[6] Zhao X., Liew K. M., Geometrically nonlinear analysis of functionally graded shells, Int $J$ Mech Sci, 51 (2009) 131 - 144.

[7] Huang H., Han Q., Buckling of imperfect functionally graded cylindrical shells under axial compression, European J Mech A/Solids, 27 (2008) 1026 - 1036.

[8] Matsunaga H., Free vibration and stability of functionally graded circular cylindrical shells according to a 2D higher - order deformation theory, Compos Struct, 88 (2009) 519 - 531.

[9] Shen H.S, Yang J, Kitipornchai S. Postbuckling of internal pressure loaded FGM cylindrical shells surrounded by an elastic medium. European J Mech A/Solids, 29 (2010) 448 - 460.

[10] Dao Huy Bich, Vu Hoai Nam, Nguyen Thi Phuong, Nonlinear postbuckling of eccentrically stiffened functionally graded plates and shallow shells, Vietnam Journal of Mechanics, 33(3) (2011) $131-147$.

[11] Duc N. D., Tung H. V., Nonlinear analysis of stability for functionally graded cylindrical panels under axial compression, Comput Mater Sci, 49 (2010) 313 - 316.

[12] Dao Van Dung, Le Kha Hoa, Nonlinear analysis of buckling and postbuckling for axially compressed functionally graded cylindrical panels with the poisson's ratio varying smoothly along the thickness, Vietnam Journal of Mechanics, 34(1) (2012) 27 - 44.

[13] Huang H., Han Q., Nonlinear elastic buckling and postbuckling of axially compressed functionally graded cylindrical shells, Int J Mech Sci, 51 (2009) 500 - 507.

[14] Huang H., Han Q., Nonlinear buckling of torsion - loaded functionally graded cylindrical shells in thermal environment, European J Mech A/Solids, 29 (2010) 42 - 48.

[15] Huang H., Han Q., Research on linear postbuckling of functionally graded cylindrical shells under radial loads, Compos Struct, 92 (2010) 1352 - 1357.

[16] Volmir A. S., Stability of elastic systems, Science Edition Moscow, (1963).

[17] Sofiyev A. H., The buckling of FGM truncated conical shells subjected to axial compressive load and resting on Winkler - Pasternak fourdations, Int J Press Piping, 87 (2010) 753 - 761.

[18] Alinia M. M., Ghanadpour SAM, Nonlinear analysis of pressure loaded FGM plates, Compos Struct, 88 (2009) 354 - 359.

[19] Brush D. D., Almroth B. O., Buckling of bars, plates and shells, MC. Graw-Hill, (1975).

Received October 19, 2011 


\section{APPENDIX}

$$
\begin{aligned}
& A_{10}= \frac{h}{2} \sum_{n \geq 0}\left(\frac{E_{m}}{n k_{1}+1}+\frac{E_{c m}}{k+n k_{1}+1}\right) c_{n} . \\
& A_{11}= \frac{h^{2}}{2} \sum_{n \geq 0}\left[E_{m}\left(\frac{1}{n k_{1}+2}-\frac{1}{2\left(n k_{1}+1\right)}\right)+E_{c m}\left(\frac{1}{k+n k_{1}+2}-\frac{1}{2\left(k+n k_{1}+1\right)}\right)\right] c_{n} . \\
& A_{12}= \frac{h^{3}}{2} \sum_{n \geq 0}\left[E_{m}\left(\frac{1}{n k_{1}+3}-\frac{1}{n k_{1}+2}+\frac{1}{4\left(n k_{1}+1\right)}\right)+\right. \\
&\left.+E_{c m}\left(\frac{1}{k+n k_{1}+3}-\frac{1}{k+n k_{1}+2}+\frac{1}{4\left(k+n k_{1}+1\right)}\right)\right] c_{n} . \\
& A_{20}= \frac{h}{2} \sum_{n \geq 0}\left[\frac{E_{m} \nu_{m}}{n k_{1}+1}+\frac{E_{m} \nu_{c m}}{(n+1) k_{1}+1}+\frac{E_{c m} \nu_{m}}{k+n k_{1}+1}+\frac{E_{c m} \nu_{c m}}{k+(n+1) k_{1}+1}\right] c_{n} . \\
& A_{21}= \frac{h^{2}}{2} \sum_{n \geq 0}\left[E_{m} \nu_{m}\left(\frac{1}{n k_{1}+2}-\frac{1}{2\left(n k_{1}+1\right)}\right)+E_{m} \nu_{c m}\left(\frac{1}{(n+1) k_{1}+2}-\frac{1}{2\left[(n+1) k_{1}+1\right]}\right)+\right. \\
&\left.+E_{c m} \nu_{m}\left(\frac{1}{k+n k_{1}+2}-\frac{1}{2\left(k+n k_{1}+1\right)}\right)+E_{c m} \nu_{c m}\left(\frac{1}{k+(n+1) k_{1}+2}-\frac{1}{2\left[k+(n+1) k_{1}+1\right]}\right)\right] c_{n} . \\
& A_{22}= \frac{h^{3}}{2} \sum_{n \geq 0}\left[E_{m} \nu_{m}\left(\frac{1}{n k_{1}+3}-\frac{1}{n k_{1}+2}+\frac{1}{4\left(n k_{1}+1\right)}\right)+E_{m} \nu_{c m}\left(\frac{1}{(n+1) k_{1}+3}-\frac{1}{(n+1) k_{1}+2}+\right.\right. \\
&+\frac{1}{4\left[(n+1) k_{1}+1\right]}+E_{c m} \nu_{m}\left(\frac{1}{k+n k_{1}+3}-\frac{1}{k+n k_{1}+2}+\frac{1}{4\left(k+n k_{1}+1\right)}\right)+ \\
&\left.+E_{c m} \nu_{c m}\left(\frac{1}{k+(n+1) k_{1}+3}-\frac{1}{k+(n+1) k_{1}+2}+\frac{1}{4\left[k+(n+1) k_{1}+1\right]}\right)\right] c_{n} . \\
& E_{3}= \frac{E_{m} h^{3}}{12}+E_{c m} h^{3}\left[\frac{1}{k+3}-\frac{1}{k+2}+\frac{1}{4 k+4}\right], \quad D=\frac{E_{1} E_{3}-E_{2}^{2}}{E_{1}\left(1-\nu^{2}\right)} . \\
& E_{30}= \frac{1}{2}\left(A_{10}-A_{20}\right), \quad A_{31}=\frac{1}{2}\left(A_{11}-A_{21}\right), \quad A_{32}=\frac{1}{2}\left(A_{12}-A_{22}\right), \\
& a_{n}= \frac{\nu_{c m}^{n}}{\left(1-\nu_{m}\right)^{n+1}}, \quad c_{n}=\left[\frac{1}{\left(1-\nu_{m}\right)^{n+1}}+\frac{1}{\left(1+\nu_{m}\right)^{n+1}}\right] \nu_{c m} . \\
& E_{c m} h
\end{aligned}
$$

